\title{
Elasticity of neck muscles in cervicogenic headache
}

\author{
Zuzana Sedlackova ${ }^{\mathrm{a}, \mathrm{b}}$, Martin Vita ${ }^{\mathrm{a}}$, Jan Hermanc, Tomas Furst' ${ }^{\mathrm{a}}$, Tomas Dornak ${ }^{\mathrm{d}}$, Miroslav Herman ${ }^{\mathrm{a}}$
}

\begin{abstract}
Aim. To compare the elasticity of the sternocleidomastoid and trapezius muscles in patients with cervicogenic headache and in healthy volunteers.

Methods. The medical history of 23 patients with cervicogenic headache was taken with a focus on pain characteristics. Elasticity of the sternocleidomastoid and trapezius muscles was measured by using shear wave elastography. Results were then compared with 23 healthy volunteers.

Results. The sternocleidomastoid muscle was significantly stiffer in patients with cervicogenic headache compared to healthy volunteers. The stiffness increased gradually from the parasternal area, where it was negligible, to the area near the mastoid process where it reached over $20 \mathrm{kPa}$. There was no difference in the stiffness of the trapezius muscle. The stiffness of the sternocleidomastoid muscle does show a significant dependence on headache characteristics (e.g., laterality, severity, or frequency).

Conclusion. The results of this pilot study show that patients with cervicogenic headache have a higher stiffness of the sternocleidomastoid muscle than healthy volunteers. These findings suggest that elastography could be used as a diagnostic tool in cervicogenic headache.
\end{abstract}

Key words: shear wave elastography, cervicogenic headache, trapezius muscle, sternocleidomastoid muscle

Received: January 15, 2021; Revised: April 26, 2021; Accepted: April 30, 2021; Available online: May 18, 2021

https://doi.org/10.5507/bp.2021.030

(c) 2022 The Authors; https://creativecommons.org/licenses/by/4.0/

${ }^{a}$ Department of Radiology, Faculty of Medicine and Dentistry, Palacky University Olomouc and University Hospital Olomouc, I. P. Pavlova 6, Olomouc, Czech Republic

${ }^{b}$ Department of Radiological Methods, Faculty of Health Sciences, Palacky University Olomouc and University Hospital Olomouc, I. P. Pavlova 6, Olomouc, Czech Republic

'Department of Otorhinolaryngology, Faculty of Medicine and Dentistry, Palacky University Olomouc and University Hospital Olomouc, I. P. Pavlova 6, Olomouc, Czech Republic

${ }^{d}$ Department of Neurology, Faculty of Medicine and Dentistry, Palacky University Olomouc and University Hospital Olomouc, I. P. Pavlova 6, Olomouc, Czech Republic

Corresponding author: Jan Herman, e-mail:jan.herman@fnol.cz

\section{INTRODUCTION}

Our work is a pilot study in patients with cervicogenic headache measuring the elasticity of the sternocleidomastoid and trapezius muscles with ultrasound shear wave elastography. This method enables non-invasive, real-time evaluation of tissue elasticity with quantitative measurements.

Almost half of the population has recurrent headaches of various aetiology ${ }^{1}$. Tension headaches, cluster headaches, and migraines are very frequent. Except for primary headaches, the pain has an external cause; an example of such a secondary headache is cervicogenic headache ${ }^{2}$.

The International Headache Society defines cervicogenic headache as a pain associated with clinical and/ or imaging evidence of a disorder or lesion within the cervical spine or soft tissues of the neck, known to be able to cause headache. The causation is demonstrated by at least two of the following: 1. headache has developed in temporal relation to the onset of the cervical disorder or appearance of the lesion; 2 . headache has significantly improved or resolved in parallel with improvement in or resolution of the cervical disorder or lesion; 3. cervical range of motion is reduced and headache is made significantly worse by provocative manoeuvres; 4 . headache is abolished following diagnostic blockade of a cervical structure or its nerve supply. And finally, the pain is not better accounted for by another diagnosis ${ }^{3}$.

The prevalence of cervicogenic headache in the general population is estimated to be between $0.4 \%$ and $2.5 \%$, but in pain management clinics, the prevalence is as high as $20 \%$ of patients with chronic headache 4 . It is often linked with functional pathologies, such as joint dysfunction, muscle stiffness, or presence of trigger points ${ }^{5-8}$. (A trigger point is a hyperirritable spot in a skeletal muscle that is associated with a hypersensitive palpable nodule in a taut band. The spot is painful on compression and can give rise to a characteristic referred pain, referred tenderness, motor dysfunction, and autonomic phenomena ${ }^{6}$.)

Sometimes, it can be hard to differentiate between the various types of headaches, and even concurrent headache types may be present. Several methods may aid in differentiating them from each other after clinical examination. Besides computed tomography or magnetic resonance imaging of the brain, ultrasonography of the neck muscles can be performed, with B-mode for imaging 
soft tissues and elastography for evaluating the stiffness of muscles. If there is suspicion of higher muscle tension from palpation, this method may confirm it $^{5-7}$.

There are several muscles that are often affected in cervicogenic headache, such as the sternocleidomastoid muscle, upper trapezius, levator scapulae, scalenes, suboccipitals, pectoralis minor, and pectoralis major ${ }^{9-10}$. We chose to examine the sternocleidomastoid and trapezius muscles because they can be easily evaluated by using ultrasonography.

\section{MATERIALS AND METHODS}

This study was approved by the Ethics Committee of Palacky University Olomouc under the reference number 153/13 and all participants have given their informed consent. We examined 46 subjects, comprising 23 patients with cervicogenic headache and 23 healthy volunteers as a control group.

The inclusion criteria in the group of patients were based on the definition of cervicogenic headache in the

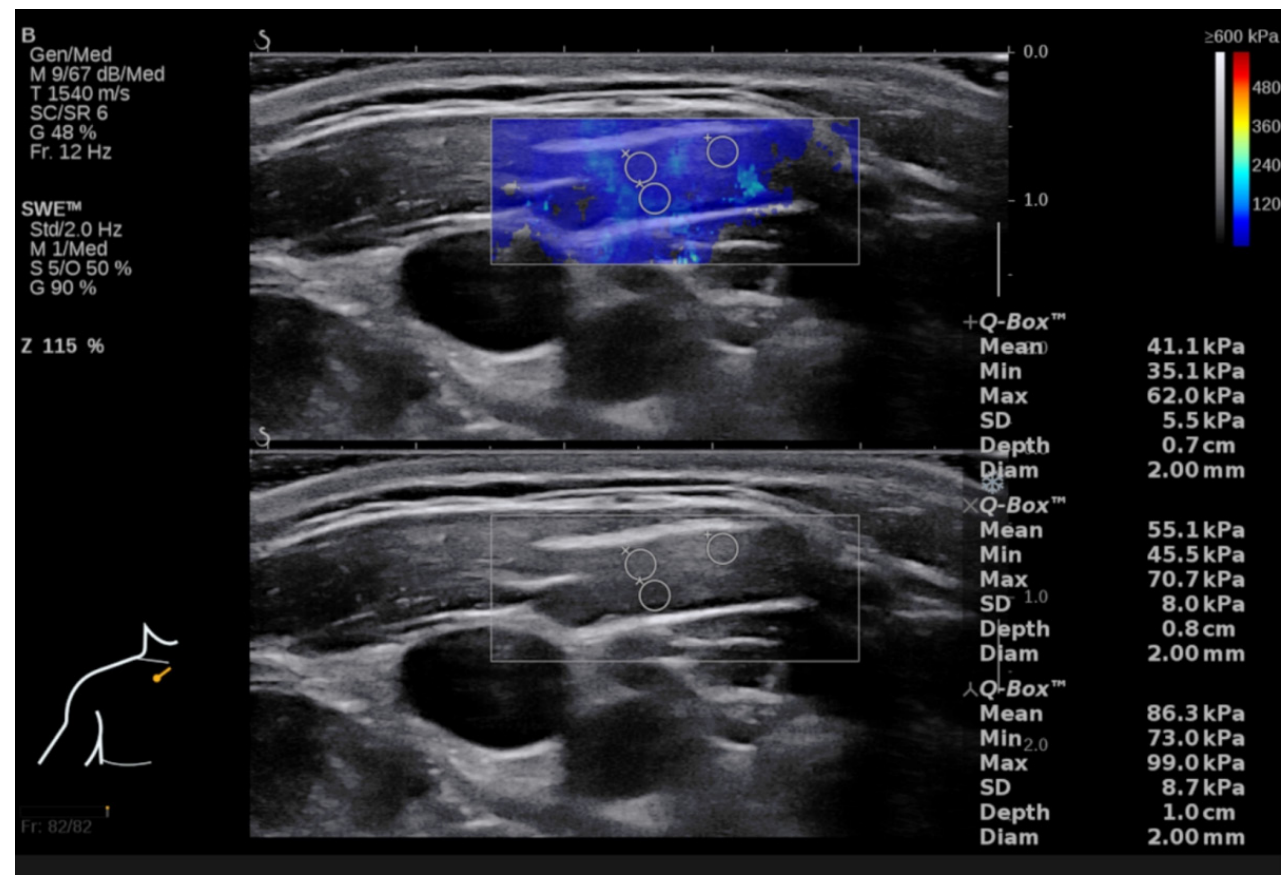

Fig. 1. Measuring stiffness of the sternocleidomastoid muscle with a linear probe in a patient with cervicogenic headache; the image depicts three ROIs at one of the four levels measured.

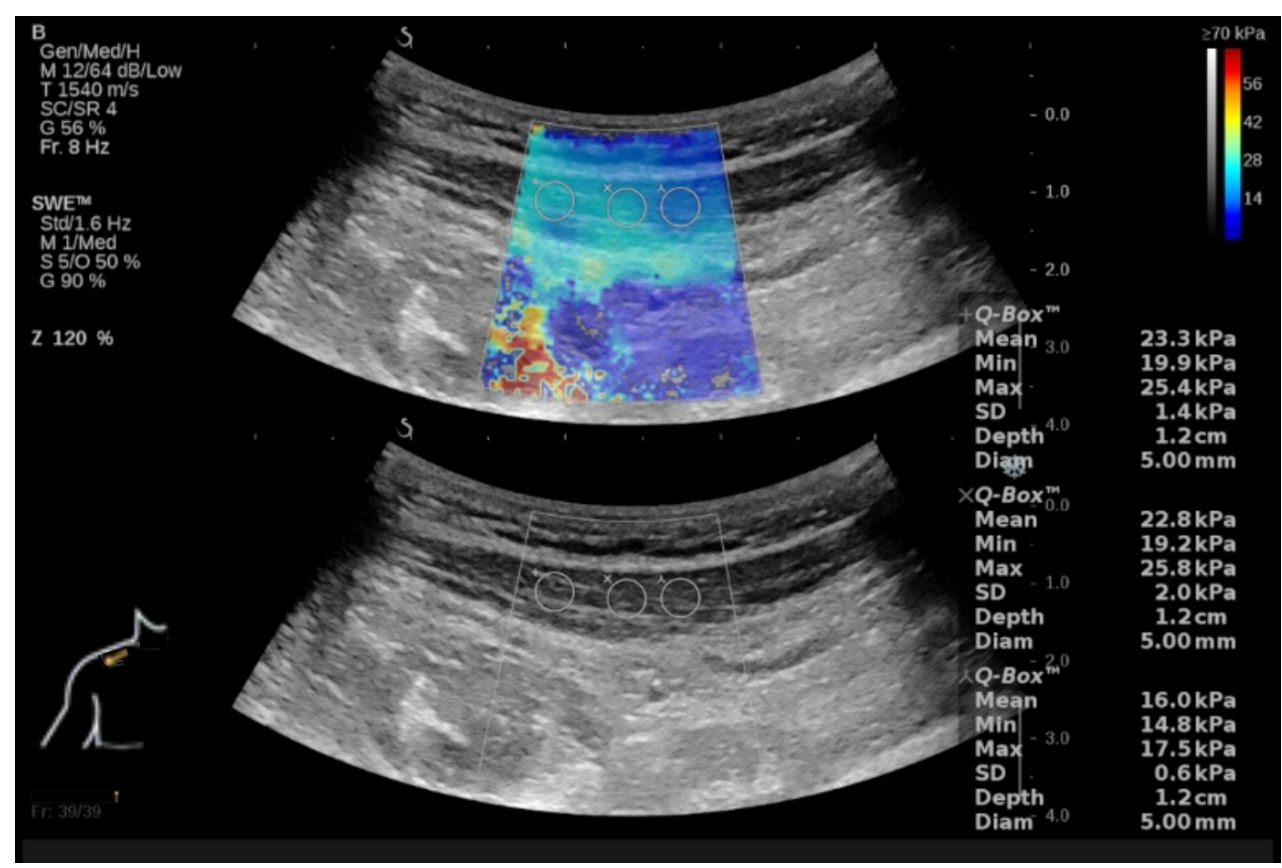

Fig. 2. Measuring stiffness of the trapezius muscle with a convex probe in a healthy volunteer. The image depicts three ROIs in one of the two assessments of the trapezius muscle. 


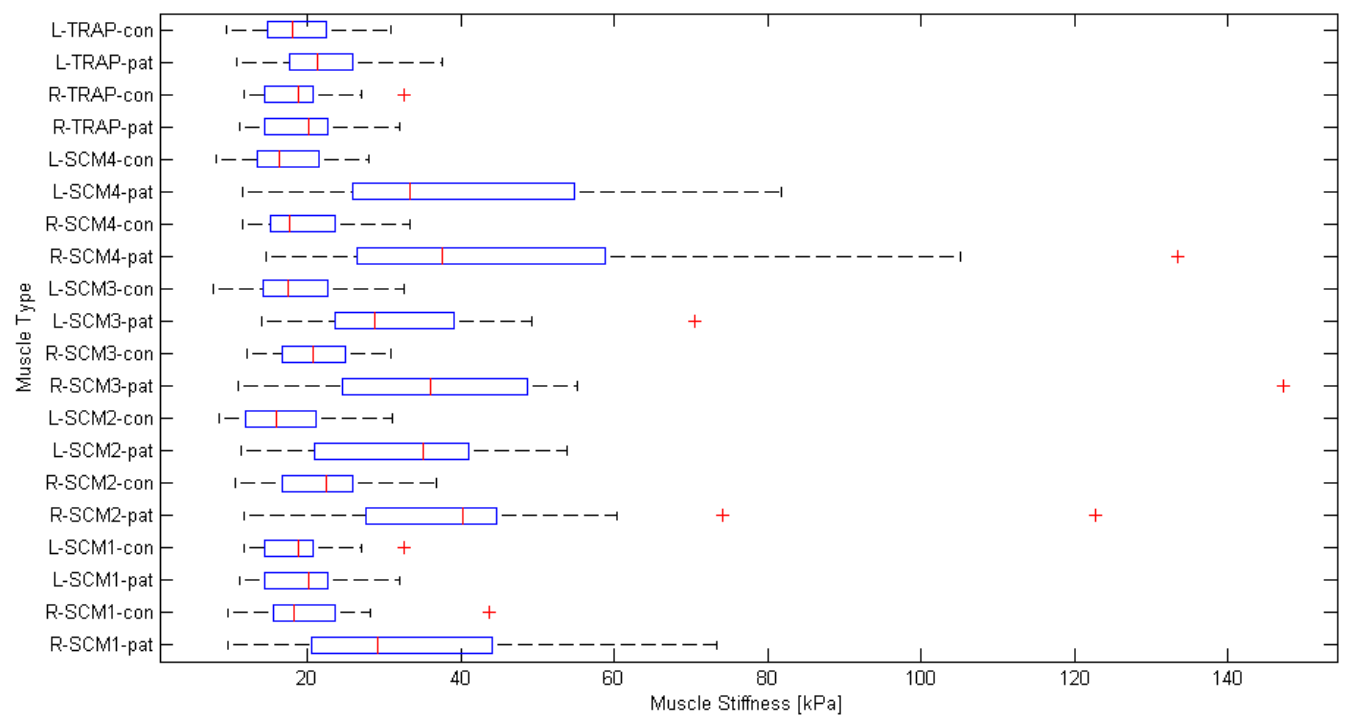

Fig. 3. An overview of the distribution of muscle stiffness values. Left-hand side (L) and right-hand side (R), patients (pat) and controls (con); the four locations at the sternocleidomastoid muscle (SCM) are described in the Introduction section; TRAP stands for the trapezius muscle. The red line denotes the median, boxes extend from $25 \%$ to $75 \%$ quantiles, and outliers are marked by a cross.

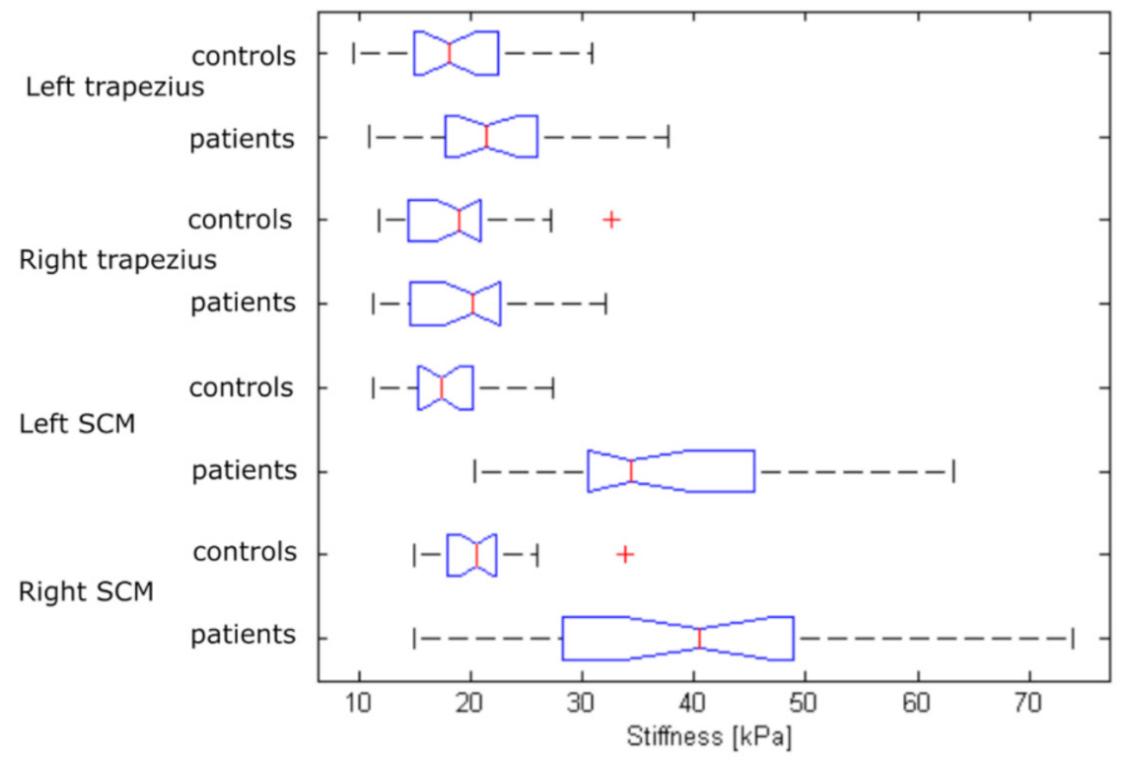

Fig. 4. A less detailed overview of the distribution of muscle stiffness values. In the case of the sternocleidomastoid muscle (SCM), the number is an average of the four medians shown in Fig. 3.

International Classification of Headache Disorders 3 (see the Introduction section above). The exclusion criteria were: age under 18 or over 55 years and another, more dominant type of headache. We excluded patients with phonophobia, photophobia, and aura.

The age of the patients ranged from 22 to 51 years (mean, 36.4 years). The patient group consisted of 20 women and 3 men. The control group did not statistically significantly differ in sex $(P=1)$, age $(P=0.8)$, or BMI $(P=0.2)$. All examined people had normal value of BMI (between 18.5-24.9).
The patients with cervicogenic headache were inquired about the characteristics of their pain. Laterality, frequency, duration of pain, character, intensity at the moment of examination and at the maximum level were all noted. We also asked whether limited head rotation, tinnitus, dizziness, nausea, vomiting, pain in the teeth or ears, and upper limb paraesthesia were present during the pain episode.

All measurements were performed by a radiologist experienced in sonoelastography using the SuperSonic Aixplorer ultrasound machine (SuperSonic Imagine, 
Table 1. An overview of the stiffnesses of all the muscles measured.

\begin{tabular}{lcccc}
\hline & $\begin{array}{c}\text { Patients, } \\
\text { right-hand side }\end{array}$ & $\begin{array}{c}\text { Patients, } \\
\text { left-hand side }\end{array}$ & $\begin{array}{c}\text { Controls, } \\
\text { right-hand side }\end{array}$ & $\begin{array}{c}\text { Controls, } \\
\text { left-hand side }\end{array}$ \\
\hline $\begin{array}{l}\text { Sternocleidomastoid, } 1 \\
\text { (parasternal area) }\end{array}$ & 34.6 & 19.8 & 20 & 18.8 \\
$\begin{array}{l}\text { Sternocleidomastoid, } 2 \\
\text { Sternocleidomastoid, 3 }\end{array}$ & 42.3 & 33.2 & & 17.4 \\
Sternocleidomastoid, 4 & 40.4 & 33.4 & 21.7 & 19 \\
(near mastoid process) & 46.8 & 20 & 39.8 & 16.9 \\
Trapezius & 19.8 & 21.9 & 18.8 & 19.1 \\
\hline
\end{tabular}

Each cell represents an average of the 23 muscle stiffness values of each subject. Muscle stiffness of each subject is the median of six repeated measurements of the average stiffness of an ROI (see the Methods section).

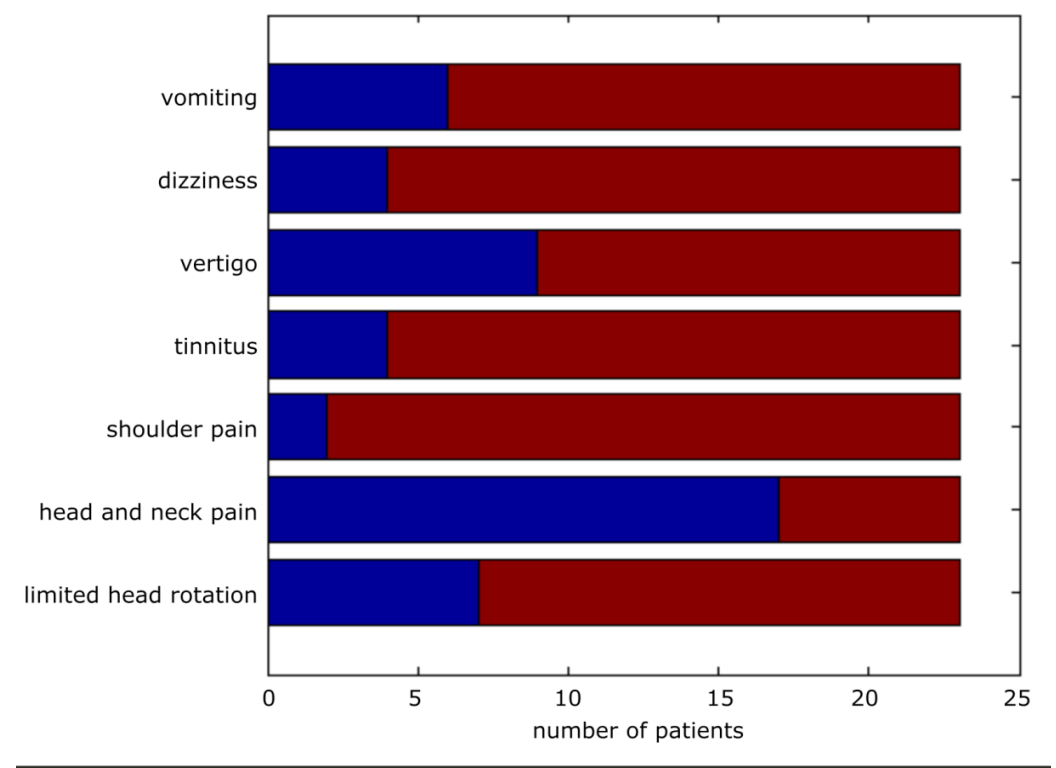

Fig. 5. The distribution of additional adverse effects in the population suffering from cervicogenic headache.

Aix-en-Provence, France) with a linear array transducer SL15-4 for the sternocleidomastoid muscle and convex transducer XC6-1 for the trapezius muscle, because the measurements with linear probe in pilot examinations were on the border of proper imaging due to bigger distance from the surface. (These examinations were excluded from the study). Elasticity of the sternocleidomastoid and trapezius muscles was measured and compared between patients and healthy volunteers. Elasticity was measured in $\mathrm{kPa}$, with higher values indicating higher stiffness (i.e., lower elasticity).

We chose to measure the sternocleidomastoid muscle at four different locations (with the muscle approximately divided into quarters, starting near the sternum, avoiding the pulsation of the carotid artery, and ending behind the ear), with six repeated measurements at each site. The accessible upper part of the trapezius was also measured six times. Since both sides were assessed, there were 60 elastographic measurements for every subject investigated. All subjects were examined in a relaxed supine position with their hands placed beside their thighs. Elasticity was measured using the region of interest (ROI) of a size of $2 \mathrm{~mm}$ in the case of the sternocleidomastoid muscle (Fig. 1) and of $5 \mathrm{~mm}$ in the trapezius muscle (Fig. 2). These values were chosen to avoid artefacts in the elastograms. We measured the maximum, mean, and minimum elasticity; however, we only used the mean values for comparison. Since the six values of the mean elasticity of each muscle often exhibited considerable variability in a single patient, we chose to calculate the median of the six values for comparison among patients.

Muscle elasticities were compared between groups of patients and healthy volunteers by the Kruskal-Wallis test. All the tests were performed in STATISTICA, version 10.0, Statsoft Inc, Tulsa, CA, and MatLab R2013b, The MathWorks Inc., Natick, MA. The level of significance was set at 0.05 .

\section{RESULTS}

Fig. 3 and 4 and Table 1 provide an overview of the results. There was a statistically significant difference in the stiffness of the sternocleidomastoid muscle between patients and healthy control group, with $P<0.001$ both on the left-hand and right-hand sides. The difference in ster- 
nocleidomastoid muscle stiffness between patients and controls increased gradually from the parasternal area, where it was negligible, to the area near the mastoid process where it reached over $20 \mathrm{kPa}$. This is more than a $100 \%$ increase with respect to the healthy volunteers. The stiffness of the trapezius muscle was not statistically significantly different between patients and controls $(P=0.58$ on the right-hand side and $P=0.15$ on the left-hand side). Out of 23 patients, nine had a more pronounced pain on the right-hand side, three on the left-hand side, and eleven had bilateral pain. Four patients suffered from headache every day, seven patients approximately once per week, and another seven once per month; others less frequently. Without taking medication, the pain lasted for one or two days in most patients.

There was no clear or systematic association between any of the adverse factors in Fig. 5 with higher stiffness of any of the muscles. This was probably due to a low number of subjects.

At the time of examination, four patients suffered from headache that reached the highest pain levels they had ever felt (two patients rated it on the VAS 0-10 as 4 and another two rated it as 7). In the remaining patients, it was lower than their maximum, with nine patients suffering from no pain at the time of examination.

Subsequently, the patients were divided according to pain intensity at the time of examination into two groups of approximately similar size $(<4$ and $\geq 4$ on the Visual Analog Scale), with 13 patients in the first group and 10 in the second one. In the group with more severe pain, four patients suffered from right-sided pain only, and the rest from bilateral pain. There was no association between the severity of the pain and the stiffness of any of the muscles measured.

\section{DISCUSSION}

We assessed the stiffness of the sternocleidomastoid and trapezius muscles in cervicogenic headache. This issue has not been studied widely so far; we found only one study that measured muscle stiffness in the same muscles, but the authors have not correlated the results with headaches. The authors of that study concluded that shear wave elastography can be a method used to evaluate muscle stiffness ${ }^{11}$. We go further and suggest that ultrasound elastography can be used to diagnose cervicogenic headache since the stiffness of the sternocleidomastoid muscle was significantly higher in the patient group than in the control one. We found no correlation of muscle stiffness with other subjective adverse factors, which may be due to a relatively small number of subjects in the study.

Two studies (none using ultrasound elastography) showed a connection between higher stiffness and pain. In the first one, the authors used ischaemic compression of the sternocleidomastoid muscle in patients with cervicogenic headache to diminish pain intensity, frequency, and duration, as well as the myofascial trigger point area, and to increase pressure tolerance and pressure pain threshold $^{12,13}$. The other study used the dry needling technique in the sternocleidomastoid muscle with similar results lowering the symptoms of cervicogenic headache ${ }^{14}$. In our study, the patients with cervicogenic headache had a significantly higher stiffness of the sternocleidomastoid muscle than healthy volunteers. The stiffness of the trapezium muscle was not different.

In a study by Park et al. ${ }^{8}$, patients with cervicogenic headache had a tangibly increased tone and stiffness of the suboccipital muscles and the upper trapezius compared to healthy subjects; however, there was no significant difference in the elasticity. This corresponds with our findings concerning the trapezius muscle.

Spasticity is a form of higher muscle tension. Two recent studies focused on upper motor neuron lesion: the first one evaluated cerebral palsy ${ }^{15}$ and the other one a stroke patient ${ }^{16}$, with both successfully using shear wave elastography to obtain results. This could be very useful since higher muscle tension could previously be evaluated only by palpation which does not provide objectively quantifiable data ${ }^{17}$.

Our results show a more frequent occurrence of rightsided headaches. Headache was present bilaterally in most patients, but in unilateral pain, the right-hand side was affected more often. This could be related to the dominance of the right upper extremity and increased upper trapezius muscle tension in pathological movement stereotypes. But this count may also have been affected by a relatively small study sample.

Cervicogenic headache is a diagnosis of exclusion, and two of the four diagnostic criteria include the success of therapeutic intervention ${ }^{3}$, which, however, is essentially done after a diagnostic conclusion. A quantitative measurement of muscle stiffness using elastography could be of help as one of the criteria for the differential diagnosis of headaches and as a method of comparison of the effect of rehabilitation and physical therapy which are usually recognised as the most effective therapies of cervicogenic headache.

The PREEMPT study by Aurora et al. suggested a paradigm of administration of onabotulinum toxin A into head and neck muscles in patients with chronic migraine that is still used ${ }^{18-20}$. It would be advisable to compare muscle stiffness in various types of headache as well as to assess more muscles, such as suboccipital ones and other described in the PREEMPT study, since a precise evaluation of their stiffness may result in a more targeted application of botulinum toxin.

\section{CONCLUSION}

Stiffness of the sternocleidomastoid muscle was significantly higher in patients with cervicogenic headache than in healthy volunteers. The stiffness increased gradually from the parasternal area, where it was negligible, to the area near the mastoid process where it reached over $20 \mathrm{kPa}$. These findings suggest that elastography could be used as a diagnostic tool in cervicogenic headache. 
Acknowledgement: Supported by Internal Grants of the Palacky University IGA LF 2020/012 and IGA LF 2020/027 and by grant DRO (FNOL, 00098892) from Ministry of Health of the Czech Republic.

Author contributions: ZS: measurements, manuscript writing; MV and JH: manuscript writing, recruitment of subjects; TF: statistical analysis; TD and MH: manuscript revisions.

Conflict of interest statement: None declared.

\section{REFERENCES}

1. Stovner L, Hagen K, Jensen R, Katsarava Z, Lipton R, Scher A, Steiner TJ, Zwart JA. The global burden of headache: a documentation of headache prevalence and disability worldwide. Cephalalgia 2007;27(3):193-210.

2. Ahmed F. Headache disorders: Differentiating and managing the common subtypes. Br J Pain 2012;6:124-32.

3. Olesen J, Dodick D, Ducros A, Evers S, First M, Goadsby P, Terwindt G. Headache Classification Committee of the International Headache Society (IHS) The International Classification of Headache Disorders, 3rd edition. Cephalalgia 2018;38(1):1-211.

4. Haldeman S, Dagenais S. Cervicogenic headaches: a critical review. Spine J 2001;(1)31-46.

5. Biondi DM. Cervicogenic Headache: A Review of Diagnostic and Treatment Strategies. The J Am Osteopath Assoc 2005;105(4):16-22.

6. Simons DG, Travell JG, Simons LS. Travell \& Simons' myofascial pain and dysfunction: the trigger point manual. Baltimore: Williams \& Wilkins. 1999.

7. Nicholson GG, Gaston J. Cervical Headache. J Orthop Sports Phys Ther 2001;31(4):184-93.

8. Park SK, Yang DJ, Kim JH, Heo JW, Uhm YH, Yoon JH. Analysis of mechanical properties of cervical muscles in patients with cervicogenic headache. J Phys Ther Sci 2017;29(2):332-35.

9. Jull G, Barrett C, Magee R, Ho P. Further clinical clarification of the muscle dysfunction in cervical headache. Cephalalgia 1999;19(3):179-85.
10. Zito G, Jull G, Story I. Clinical tests of musculoskeletal dysfunction in the diagnosis of cervicogenic headache. Man Ther 2006;11(2):11829.

11. Ewertsen C, Carlsen J, Perveez MA, Schytz H. Reference Values for Shear Wave Elastography of Neck and Shoulder Muscles in Healthy Individuals. Ultrasound Int Open 2018;4(1):23-29.

12. Jafari M, Bahrpeyma F, Mokhtari-Dizaji M, Nasiri A. Novel method to measure active myofascial trigger point stiffness using ultrasound imaging. J Bodyw Mov Ther 2018;22(2):374-78.

13. Jafari M, Bahrpeyma F, Togha M. Effect of ischemic compression for cervicogenic headache and elastic behavior of active trigger point in the sternocleidomastoid muscle using ultrasound imaging. J Bodyw Mov Ther 2017;21:933-39.

14. Martín-Rodríguez A, Sáez-Olmo E, Pecos-Martín D, Calvo-Lobo C Effects of dry needling in the sternocleidomastoid muscle on cervical motor control in patients with neck pain: a randomised clinical trial. Acupunc Med 2019;37(3):151-63.

15. Brandenburg J, Eby S, Song P, Kingsley-Berg S, Bamlet W, Sieck G, An KN. Quantifying passive muscle stiffness in children with and without cerebral palsy using ultrasound shear wave elastography. Dev Med Child Neurol 2016;58(12):1288-94.

16. Eby SF, Zhao H, Song P, Vareberg BJ, Kinnick RR, Greenleaf JF, An KN, Brown AW, Chen S. Quantifying spasticity in individual muscles using shear wave elastography. Radiol Case Rep 2017;12(2):348-52.

17. Rathbone A, Grosman-Rimon L, Kumbhare D. Interrater Agreement of Manual Palpation for Identification of Myofascial Trigger Points: A Systematic Review and Meta-Analysis. Clin J Pain 2017;33(8):715-29.

18. Aurora SK, Dodick DW, Diener H-C, DeGryse RE, Turkel C, Lipton RB, Silberstein SD. Onabotulinumtoxin A for chronic migraine: efficacy, safety, and tolerability in patiens who received all five treatment cycles in the PREEMPT clinical program. Acta Neurol Scand 2014;129:61-70.

19. Aurora SK, Dodick DW, Turkel CC, DeGryse RE, Silberstein SD, Lipton RB, Diener HC, Brin MF. OnabotulinumtoxinA for treatment of chronic migraine: results from the double-blind, randomized, placebo-controlled phase of the PREEMPT 1 trial. Cephalalgia 2010;30:793-803.

20. Aurora SK, Gawel M, Brandes JL, Pokta S, Vandenburgh AM Botulinum toxin type a prophylactic treatment of episodic migraine: a randomized, double-blind, placebo-c ontrolled exploratory study. Headache 2007;47(4):486-99. 